\title{
Pacific
}

Journal of

Mathematics

\section{INTERPOLATED FREE GROUP FACTORS}

KENNETH JAY DyKeMA 


\section{INTERPOLATED FREE GROUP FACTORS}

\section{Ken DYKema}

The interpolated free group factors $L\left(\mathbf{F}_{r}\right)$ for $1<r \leq \infty$ (also defined by $F$. Rădulescu) are given another (but equivalent) definition as well as proofs of their properties with respect to compression by projections and free products. In order to prove the addition formula for free products, algebraic techniques are developed which allow us to show $R * R \cong L\left(\mathrm{~F}_{2}\right)$ where $R$ is the hyperfinite $\mathrm{II}_{1}$-factor.

Introduction. The free group factors $L\left(\mathbf{F}_{n}\right)$ for $n=2,3, \ldots, \infty$ (introduced in [4]) have recently been extensively studied [11, 2, 5, $6,7]$ using Voiculescu's theory of freeness in noncommutative probability spaces (see $[8,9,10,11,12,13]$, especially the latter for an overview). One hopes to eventually be able to solve the isomorphism question, first raised by R. V. Kadison of whether $L\left(\mathbf{F}_{n}\right) \cong L\left(\mathbf{F}_{m}\right)$ for $n \neq m$. In [7], F. Rădulescu introduced $\mathrm{II}_{1}$-factors $L\left(\mathbf{F}_{r}\right)$ for $1<$ $r \leq \infty$, equalling the free group factor $L\left(\mathbf{F}_{n}\right)$ when $r=n \in \mathbf{N} \backslash\{0,1\}$ and satisfying

$$
L\left(\mathbf{F}_{r}\right) * L\left(\mathbf{F}_{r^{\prime}}\right)=L\left(\mathbf{F}_{r+r^{\prime}}\right) \quad\left(1<r, r^{\prime} \leq \infty\right)
$$

and

$$
L\left(\mathbf{F}_{r}\right)_{\gamma}=L\left(\mathbf{F}\left(1+\frac{r-1}{\gamma^{2}}\right)\right) \quad(1<r \leq \infty, 0<\gamma<\infty),
$$

where for a $\mathrm{II}_{1}$-factor $\mathscr{M}, \mathscr{M}_{\gamma}$ means the algebra [4] defined as follows: for $0<\gamma \leq 1, \mathscr{M}_{\gamma}=p \mathscr{M} p$, where $p \in \mathscr{M}$ is a selfadjoint projection of trace $\gamma$; for $\gamma=n=2,3, \ldots$ one has $\mathscr{M}_{\gamma}=\mathscr{M} \otimes M_{n}(\mathbf{C})$; for $0<\gamma_{1}, \gamma_{2}<\infty$ one has

$$
\mathscr{M}_{\gamma_{1} \gamma_{2}}=\left(\mathscr{M}_{\gamma_{1}}\right)_{\gamma_{2}} \text {. }
$$

We had independently found the interpolated free group factors $L\left(\mathbf{F}_{r}\right) \quad(1<r \leq \infty)$ and the formulas (1) and (2), defining them differently and using different techniques. In this paper we give our definition and proofs. This picture of $L\left(\mathbf{F}_{r}\right)$ is sometimes more convenient, e.g. $\S 4$ of [3]. It is a natural extension of the result [2] that

$$
\mathrm{L}(\mathbf{Z}) * R \cong \mathrm{L}\left(\mathbf{F}_{2}\right),
$$


where $R$ is the hyperfinite $\mathrm{II}_{1}$-factor. We also introduce some elementary algebraic techniques for freeness which have further application in [3]. One consequence of them that we prove here is that $R * R \cong \mathrm{L}\left(\mathbf{F}_{2}\right)$.

This paper has four sections. In $\S 1$ we state a random matrix result (from [2], [7]) and some consequences; in $\S 2$ we define the interpolated free group factors and prove the formula (2); in $\S 3$ we develop the algebraic techniques; in $\S 4$ we prove the addition formula (1) and also make an observation from (1) and (2) (also observed in [7]) that, as regards the isomorphism question, we must have one of two extremes. Our original proof of the addition formula (1) was a fairly messy application of the algebraic techniques developed in $\S 3$. The proof of Theorem 4.1 that appears here, while still using the algebraic techniques in an essential way, benefits significantly from ideas found in the proof of F. Rădulescu [7].

1. The matrix model. Voiculescu, as well as developing the whole notion of freeness in noncommutative probability spaces, had the fundamental idea of using Gaussian random matrices to model freeness, which he developed in [12]. In [2], we extended this matrix model to the non-Gaussian case and also to be able to handle semicircular families together with a free finite dimensional algebra. As Rădulescu observed in [7], the matrix model necessary to be able to handle the free finite dimensional algebra can be easily proved in the Gaussian case directly using Voiculescu's methods (cf. the appendix of [2]). In any case, we shall use this matrix model in this paper, and quote it here, as well as some results of it. Our notation for random matrices will be as in [2]. A trivial reformulation of Theorem 2.1 of [2] gives

THEOREM 1.1. Let $Y(s, n) \in M_{n}(L)$ for $s \in S$ be selfadjoint independently distributed $n \times n$ random matrices as in Theorem 2.1 of [2]. For

$$
c=\left(\begin{array}{ccc}
c_{11} & \ldots & c_{1 N} \\
\vdots & \ddots & \vdots \\
c_{N 1} & \ldots & c_{N N}
\end{array}\right) \in M_{N}(\mathbf{C})
$$

and for $n$ a multiple of $N$ let

$$
c(n)=\left(\begin{array}{ccc}
c_{11} I_{n / N} & \ldots & c_{1 N} I_{n / N} \\
\vdots & \ddots & \vdots \\
c_{N 1} I_{n / N} & \ldots & c_{N N} I_{n / N}
\end{array}\right)
$$


be a constant matrix in $M_{n}(L)$. Then

$$
\left\{(\{Y(s, n)\})_{s \in S},\left\{c(n) \mid c \in M_{N}(\mathbf{C})\right\}\right\}
$$

is an asymptotically free family as $n \rightarrow \infty$, and each $Y(s, n)$ has for limit distribution a semicircle law.

An immediate result of the above is (3.2 of [2]):

THEOREM 1.2. In a noncommutative probability space $(\mathscr{M}, \phi)$ with $\phi$ a trace, let $\nu_{1}=\left\{X^{s} \mid s \in S\right\}$ be a semicircular family and let $\nu_{2}=$ $\left\{e_{i j} \mid 1 \leq i, j \leq n\right\}$ be a system of matrix units such that $\left\{\nu_{1}, \nu_{2}\right\}$ is free. Then in $\left(e_{11} \mathscr{M} e_{11},\left.n \phi\right|_{e_{11}} \mathscr{M} e_{11}\right), \omega_{1}=\left\{e_{1 i} X^{s} e_{i 1} \mid 1 \leq i \leq n, s \in\right.$ $S\}$ is a semicircular family and $\omega_{2}=\left\{e_{1 i} X^{s} e_{j 1} \mid 1 \leq i<j \leq n, s \in S\right\}$ is a circular family such that $\left\{\omega_{1}, \omega_{2}\right\}$ is free.

The following is analogous to Theorem 2.4 of [11].

THEOREM 1.3. In a noncommutative probability space $(\mathscr{M}, \phi)$ with $\phi$ a trace, let $\nu=\left\{X^{s} \mid s \in S\right\}$ be a semicircular family and let $R$ be a copy of the hyperfinite $\mathrm{II}_{1}$-factor such that $\{\nu, R\}$ is free. Let $p \in R$ be a nonzero selfadjoint projection. Then in $\left(p \mathscr{M} p,\left.\phi(p)^{-1} \phi\right|_{p \mathscr{M}_{p}}\right)$, $\omega=\left\{p X^{s} p \mid s \in S\right\}$ is a semicircular family and $\{p R p, \omega\}$ is free. (Note from [4] that $p R p$ is also a copy of the hyperfinite $\mathrm{II}_{1}$-factor.)

Proof. Suppose first that $\phi(p)=m / 2^{k}$, a dyadic rational number. Since for $U \in R$ a unitary, $\left\{R, U \nu U^{*}\right\}$ is free, we may let $p$ be any projection in $R$ of the given trace. Writing $R=M_{2^{k}} \otimes M_{2} \otimes$ $M_{2} \otimes \cdots$, we use Theorem 1.1 in order to model $\nu$ as the limit of selfadjoint independently distributed random matrices of size $n=$ $2^{k}, 2^{k+1}, 2^{k+2}, \ldots$, and model a dense subalgebra of $R$ (equal to the tensor product of matrix algebras) by constant random matrices. Choosing $p$ to correspond to a diagonal element of $M_{2^{k}}$, we may apply Theorem 1.1 again to see that $\omega$ is a semicircular family, $p R p \cong$ $M_{m} \otimes M_{2} \otimes M_{2} \otimes \cdots$, and $\{p R p, \omega\}$ is free.

Now for general $p$, let $\left(p_{l}\right)_{l=1}^{\infty}$ be a decreasing sequence of projections in $R$ which converge to $p$ and such that each $\phi\left(p_{l}\right)$ is a dyadic rational number. Then

$$
\left\{p_{l} R p_{l}=\left\{p_{l} y p_{l} \mid y \in R\right\},\left\{p_{l} X^{s} p_{l} \mid s \in S\right\}\right\}
$$

has limit distribution equal to $\{p R p, \omega\}$ as $l \rightarrow \infty$. For each $l$ we have freeness and semicircularity, hence also in the limit.

In addition, modeling $R$ and a semicircular family as in the above proof, we can easily prove 
THEOREM 1.4. In a noncommutative probability space $(\mathscr{M}, \phi)$ with $\phi$ a trace, let $\nu=\left\{X^{s} \mid s \in S\right\}$ be a semicircular family, and let $R$ be a hyperfinite $\mathrm{II}_{1}$-factor containing a system of matrix units $\left\{e_{i j} \mid 1 \leq\right.$ $i, j \leq n\}$, such that $\{\nu, R\}$ is free. Then in $\left(e_{11} \mathscr{M} e_{11},\left.n \phi\right|_{e_{11} \mathscr{M} e_{11}}\right)$, $\omega_{1}=\left\{e_{1 i} X^{s} e_{i 1} \mid 1 \leq i \leq n, s \in S\right\}$ is a semicircular family and $\omega_{2}=\left\{e_{1 i} X^{s} e_{j 1} \mid 1 \leq i<j \leq n, s \in S\right\}$ is a circular family such that $\left\{\omega_{1}, \omega_{2}, e_{11} R e_{11}\right\}$ is *-free.

\section{Definition and compressions of $L\left(\mathbf{F}_{r}\right)$.}

Definition 2.1. In a $\mathrm{W}^{*}$-probability space $(\mathscr{M}, \tau)$, where $\tau$ is a faithful trace, let $R$ be a copy of the hyperfinite $\mathrm{II}_{1}$-factor and $\omega=\left\{X^{t} \mid t \in T\right\}$ be a semicircular family such that $R$ and $\omega$ are free. Then $L\left(\mathbf{F}_{r}\right)$ for $1<r \leq \infty$ will denote any factor isomorphic to $\left(R \cup\left\{p_{t} X^{t} p_{t} \mid t \in T\right\}\right)^{\prime \prime}$, where $p_{t} \in R$ are selfadjoint projections and $r=1+\sum_{t \in T} \tau\left(p_{l}\right)^{2}$.

Proposition 2.2. $L\left(\mathbf{F}_{r}\right)$ is well-defined, i.e. if

$$
\mathscr{A}=\left(R \cup\left\{p_{t} X^{t} p_{t} \mid t \in T\right\}\right)^{\prime \prime} \quad \text { and } \mathscr{B}=\left(R \cup\left\{q_{t} X^{t} q_{t} \mid t \in T\right\}\right)^{\prime \prime} \text {, }
$$

where $1+\sum \tau\left(p_{t}\right)^{2}=r=1+\sum \tau\left(q_{t}\right)^{2}$, then $\mathscr{A} \cong \mathscr{B}$.

Proof. We show that $\mathscr{A}$ (and thus also $\mathscr{B}$ ) is isomorphic to an algebra of a certain "standard form." Let $\left(f_{k}\right)_{k=1}^{\infty}$ be an orthogonal family of projections in $R$ such that $\tau\left(f_{k}\right)=2^{-k}$, and let $f_{0}=1$. If $r<\infty$ let $N_{l} \quad(l \geq 0)$ be nonnegative integers corresponding to the base 4 expansion for $r$, i.e. $r=\sum_{l=0}^{\infty} N_{l} 4^{-l}, N_{l} \leq 3$ if $l \geq 1$ and $\sum_{l>l^{\prime}} N_{l} 4^{-l}<4^{-l^{\prime}} \forall l^{\prime} \geq 0$. If $r=\infty$ we let $N_{0}=\infty$ and $N_{l}=0 \forall l \geq 1$. Let $S \subseteq T, k_{s} \in \mathbf{N}=\{0,1,2, \ldots\}$ for $s \in S$ be such that $\left|\left\{s \in S \mid k_{s}=l\right\}\right|=N_{l} \forall l \geq 0$. The algebra of standard form is then $\mathscr{C}=\left(R \cup\left\{f_{k_{s}} X^{s} f_{k_{s}} \mid s \in S\right\}\right)^{\prime \prime}$. Showing $\mathscr{A} \cong \mathscr{C}$ will prove the proposition.

Proving $\mathscr{A} \cong \mathscr{C}$ is an exercise in cutting and pasting. Note that if $U_{t}$ are unitaries in $R(t \in T)$, then $\left\{R,\left(\left\{U_{t} X^{t} U_{t}^{*}\right\}\right)_{t \in T}\right\}$ is free in $(\mathscr{M}, \tau)$. Moreover, each projection $p \in R$ is conjugate by a unitary in $R$ to a projection that is a (possibly infinite) sum of projections in $\left\{f_{k} \mid k \geq 1\right\}$. Hence letting $T^{\prime}=\left\{t \in T \mid p_{t} \neq 0\right\}$, we may assume without loss of generality that each $p_{t}$ for $t \in T^{\prime}$ is equal to such a sum, and we write $p_{t}=\sum_{k \in K_{t}} f_{k}$, for $K_{t} \subseteq \mathbf{N} \backslash\{0\}$ whenever $t \in T^{\prime}$ and $p_{t} \neq 1$, and we set $K_{t}=\{0\}$ if $p_{t}=1$. Then

$$
\mathscr{A}=\left(R \cup\left\{f_{k} X^{t} f_{k^{\prime}} \mid k, k^{\prime} \in K_{t}, k^{\prime} \leq k, t \in T^{\prime}\right\}\right)^{\prime \prime} .
$$


Now we may appeal to the matrix model $(\S 1)$ to see that (enlarging $T$ if necessary),

$$
\mathscr{A}=\left(R \cup\left\{f_{k} X^{\alpha\left(k, k^{\prime}, t\right)} f_{k^{\prime}} \mid k, k^{\prime} \in K_{t}, k^{\prime} \leq k, t \in T^{\prime}\right\}\right)^{\prime \prime},
$$

where $\alpha$ is a $1-1$ map from $\left\{k, k^{\prime} \in K_{t}, k^{\prime} \leq k, t \in T^{\prime}\right\}$ onto a subset $T^{\prime \prime}$ of $T$. (The truth of the above assertion is most easily demonstrated when $T^{\prime}$ and each $K_{t}$ are finite; the general case then follows by taking inductive limits.)

Consider for a moment $f_{k} X^{t} f_{k^{\prime}}$ for $k^{\prime}<k, t \in T$. Note that $f_{k^{\prime}}$ is the sum of $2^{k-k^{\prime}}$ orthogonal projections, each of which is equivalent in $R$ to $f_{k}$. Using the matrix model shows that

$$
\begin{aligned}
& \left(R \cup\left\{f_{k} X^{t} f_{k^{\prime}}\right\}\right)^{\prime \prime} \\
& \quad \cong\left(R \cup\left\{f_{k} X^{t} f_{k} \mid 1 \leq j \leq 2^{k-k^{\prime}}\right\} \cup\left\{f_{k} X^{t^{\prime}} f_{k} \mid 1 \leq j \leq 2^{k-k^{\prime}}\right\}\right)^{\prime \prime},
\end{aligned}
$$

where $t_{1}, \ldots, t_{2^{k-k^{\prime}}}, t_{1}^{\prime}, \ldots, t_{2^{k-k^{\prime}}}^{\prime}$ are distinct elements of $T$, and the isomorphism in (4) maps $R$ identically into itself. Using inductive limits, one obtains

$$
\mathscr{A} \cong \tilde{\mathscr{C}}=\left(R \cup\left\{f_{k_{s}} X^{s} f_{k_{s}} \mid s \in S^{\prime}\right\}\right)^{\prime \prime},
$$

for $S^{\prime}$ some subset of $T, k_{s} \in \mathbf{N}$ for each $s \in S^{\prime}$. Moreover, checking the arithmetic of the above moves shows that $1+\sum_{s \in S^{\prime}} \tau\left(f_{k_{s}}\right)^{2}=r$.

Now for the pasting. Note that by the matrix model,

$$
\left(R \cup\left\{f_{k} X^{t_{i}} f_{k} \mid 1 \leq i \leq 4\right\}\right)^{\prime \prime} \cong\left(R \cup\left\{f_{k-1} X^{t} f_{k-1}\right\}\right)^{\prime \prime}
$$

by an isomorphism mapping $R$ identically to itself, whenever $k \geq$ $1, t_{1}, \ldots, t_{4}$ are distinct elements of $T$ and $t \in T$. Suppose $r<$ $\infty$. If $r$ is not a dyadic rational then for each $l \geq 0$ let $\mathscr{C}(l)=$ $\left(R \cup\left\{f_{k_{s}} X^{s} f_{k_{s}} \mid s \in S, k_{s} \leq l\right\}\right)^{\prime \prime} \subseteq \mathscr{C}$. There is an increasing sequence $S^{\prime}(l)$ of finite subsets of $S^{\prime}$ such that $\sum_{s \in S^{\prime}(l)} 4^{-k_{s}}=\sum_{0 \leq k \leq l} N_{k} 4^{-k}$ and $\bigcup_{l \geq 1} S^{\prime}(l)=S^{\prime}$. Let

$$
\widetilde{\mathscr{C}}(l)=\left(R \cup\left\{f_{k_{s}} X^{s} f_{k_{s}} \mid s \in S^{\prime}(l)\right\}\right)^{\prime \prime} \subseteq \tilde{\mathscr{C}} .
$$

Using (6) repeatedly we can find a compatible family of isomorphisms $\phi_{l}: \widetilde{\mathscr{C}}(l) \rightarrow \mathscr{C}(l)$, and taking inductive limits yields $\widetilde{\mathscr{C}} \cong \mathscr{C}$. If $r$ equals a dyadic rational and $S^{\prime}$ is finite then a finite number of applications of (6) yields $\tilde{\mathscr{C}} \cong \mathscr{C}$. If $S^{\prime}$ is infinite, let $l$ be largest such that $N_{l} \neq 0$, let $\sigma \in S$ be such that $k_{\sigma}=l$ and let $f_{l} \geq g_{l+1} \geq$ $g_{l+2} \geq \cdots$ be projections in $R$ where $\tau\left(g_{m}\right)=2^{-m}$. For $m>l$ let $\mathscr{C}(m)=\left(R \cup\left\{f_{k_{s}} X^{s} f_{k_{s}} \mid s \in S \backslash\{\sigma\}\right\} \cup\left\{f_{k_{\sigma}} X^{\sigma} f_{k_{\sigma}}-g_{m} X^{\sigma} g_{m}\right\}\right)^{\prime \prime} \subseteq \mathscr{C}$. 
Then as before we can use the matrix model to find an increasing family $\tilde{\mathscr{C}}(m)$ of subalgebras of $\widetilde{\mathscr{C}}$ whose union generates $\tilde{\mathscr{C}}$ and compatible isomorphisms $\widetilde{\mathscr{C}}(m) \rightarrow \mathscr{C}(m)$. Taking inductive limits yields $\tilde{\mathscr{C}} \cong \mathscr{C}$.

If $r=\infty$, then considering $S^{\prime}$ from (5) and letting $S_{k}^{\prime}=\{s \in$ $\left.S^{\prime} \mid k_{s}=k\right\}$, we have that $\sum_{k=0}^{\infty}\left|S_{k}^{\prime}\right| 4^{-k}=\infty$. Now by repeated application of (6), we can transform the situation (by isomorphisms mapping $R$ identically to itself) so that first some $\left|S_{k}^{\prime}\right|=\infty$, then all $\left|S_{k}^{\prime}\right|=\infty$, then $\left|S_{0}^{\prime}\right|=\infty$ and $\left|S_{k}^{\prime}\right|=0$ for all $k \geq 1$. Thus $\widetilde{\mathscr{C}}=L\left(\mathbf{F}_{\infty}\right)$ by (3).

REMARK 2.3. Formula (2), together with the fact that $L\left(\mathbf{F}_{r}\right)$ for $r \in$ $\mathbf{N}$ is the free group factor on $r$ generators, shows that Definition 2.1 is equivalent to Rădulescu's definitions 4.1 and 5.3 of [7]. However, for $r \geq 2$ (i.e. Rădulescu's 4.1), this equivalence can be seen directly using the "standard form" of $L\left(\mathbf{F}_{r}\right)$ as defined in Proposition 1.3, and by noting that the isomorphism

$$
R * L(\mathbf{Z}) \stackrel{\sim}{\rightarrow} L(\mathbf{Z}) * L(\mathbf{Z})
$$

in [2] sends the set of projections $\left\{f_{k} \mid k \geq 1\right\} \subset R$ into one of the copies of $L(\mathbf{Z})$ on the right-hand side of $(8)$.

The formula in the following theorem for the compression of an interpolated free group factor $L\left(\mathbf{F}_{r}\right)$ by a projection of trace $\gamma$ was first proved by Voiculescu [11] for the cases $r=2,3, \ldots, \gamma=$ $\frac{1}{2}, \frac{1}{3}, \frac{1}{4}, \ldots$ and $r=\infty, \gamma \in \mathbf{Q}_{+}$. It was then extended by $\mathrm{F}$. Rădulescu in [5] for $r=\infty$ and $\gamma \in \mathbf{R}_{+}$, and in [6] for $r=2,3, \ldots$ and $\gamma=\frac{1}{\sqrt{2}}, \frac{1}{\sqrt{3}}, \ldots$ Of course, Rădulescu also proved this theorem in the generality stated here in [7].

THEOREM 2.4 .

$$
L\left(\mathbf{F}_{r}\right)_{\gamma}=L\left(\mathbf{F}\left(1+\frac{r-1}{\gamma^{2}}\right)\right)
$$

for $1<r \leq \infty$ and $0<\gamma<\infty$.

Proof. It suffices to show the case $0<\gamma<1$. Let $L\left(\mathbf{F}_{r}\right)=\mathscr{A}=$ $\left(R \cup\left\{p_{t} X^{t} p_{t} \mid t \in T\right\}\right)^{\prime \prime}$ be as in Definition 2.1 , so $1+\sum_{t \in T} \tau\left(p_{t}\right)^{2}=r$. Let $p \in R$ be a projection having trace $\gamma$. Without loss of generality, we may assume that each $p_{t} \leq p$. Then

$$
p \mathscr{A} p=\left(p R p \cup\left\{p_{t} X^{t} p_{t} \mid t \in T\right\}\right)^{\prime \prime},
$$

which by Theorem 1.3 is an interpolation free group factor. Counting gives the formula (9). 
3. Algebraic techniques. A crucial ingredient of our proof of the addition formula for free products (1) will be showing that $R * R \cong$ $R * L(\mathbf{Z})$, with the isomorphism being the identity map on the first copy of $R$. In order to show this, we will introduce some elementary techniques (Definition 3.4, proof of Theorem 3.5) that are algebraic in nature. These techniques have extensive further applications to free products, as will be seen in [3].

REMARK 3.1. In this section, all von Neumann algebras will be finite and have fixed normalized faithful traces associated to them, and all isomorphisms and inclusions of von Neumann algebras will be assumed to be trace preserving. Von Neumann algebras that we obtain from others by certain operations will have associated traces given by the following conventions:

(1) group von Neumann algebras $L(G)$ for $G$ a discrete group will have their canonical traces (equal to the vector-state for the vector $\left.\delta_{e} \in l^{2}(G)\right)$;

(2) factors, such as matrix algebras $M_{n}=M_{n}(\mathbf{C})$ or the hyperfinite $\mathrm{II}_{1}$-factor $R$, will have (of course) their unique normalized traces;

(3) a tensor product $A \otimes B$ of algebras will have the tensor product trace $\tau_{A} \otimes \tau_{B}$ of the given traces on $A$ and $B$;

(4) a free product $A * B$ of algebras will have the free product trace $\tau_{A} * \tau_{B}$ of the given traces on $A$ and $B$;

(5) if $\mathscr{M}$ is a von Neumann algebra with faithful trace $\tau$, and $p$ is a projection in $\mathscr{M}$, then $p \mathscr{M} p$ will have trace $\left.\tau(p)^{-1} \tau\right|_{p \mathscr{M} p}$.

Also, if $A$ is an algebra with specific trace, $\stackrel{\circ}{A}$ will denote the ensemble of elements of $A$ whose trace is zero.

First we examine $L\left(\mathbf{Z}_{2}\right) * L\left(\mathbf{Z}_{2}\right)$ (where $\mathbf{Z}_{2}$ is the two element group). The fact that $\mathscr{M}=L\left(\mathbf{Z}_{2} * \mathbf{Z}_{2}\right) \cong L(\mathbf{Z}) \otimes M_{2}$ is well known, but we will need the following picture of $\mathscr{M}$.

Proposition 3.2. Consider $\mathscr{M}=L\left(\mathbf{Z}_{2}\right) * L\left(\mathbf{Z}_{2}\right)$ with trace $\tau$, and let $p$ and $q$ be projections of trace $\frac{1}{2}$ generating the first and respectively the second copy of $L\left(\mathbf{Z}_{2}\right)$. Then

$$
\mathscr{M} \cong L^{\infty}\left(\left[0, \frac{\pi}{2}\right], \nu\right) \otimes M_{2}
$$

where $\nu$ is a probability measure on $\left[0, \frac{\pi}{2}\right]$ without atoms and $\tau$ is given by integration with respect to $\nu$ tensored with the normalized trace on $M_{2}=M_{2}(\mathbf{C})$. Moreover, in the setup of (10), we have that 
(11) $p=\left(\begin{array}{ll}1 & 0 \\ 0 & 0\end{array}\right)$ and $q=\left(\begin{array}{cc}\cos ^{2} \theta & \cos \theta \sin \theta \\ \cos \theta \sin \theta & \sin ^{2} \theta\end{array}\right)$, where $\theta \in\left[0, \frac{\pi}{2}\right]$.

Proof. It is well known that the universal unital $C^{*}$-algebra generated by two projections $p$ and $q$ is $A=\left\{f:\left[0, \frac{\pi}{2}\right] \rightarrow M_{2}(\mathbf{C}) \mid f(0)\right.$ and $f\left(\frac{\pi}{2}\right)$ diagonal $\}$, with $p$ and $q$ as in (11). $\mathscr{M}$ thus has a dense subalgebra equal to a quotient of $A$, and $\tau$ gives a trace on $A$. One can easily see that a trace on $A$ must be of the following form. Let $f(t)_{1}$ and $f(t)_{2}$ be the diagonal values of $f(t)$ for $t=0$ or $\frac{\pi}{2}$. Then

$$
\begin{aligned}
\tau(f)= & a_{1} f(0)_{1}+a_{2} f(0)_{2}+\int_{0}^{\pi / 2} \tau_{2}(f(t)) d \nu(t) \\
& +b_{1} f\left(\frac{\pi}{2}\right)_{1}+b_{2} f\left(\frac{\pi}{2}\right)_{2},
\end{aligned}
$$

where $\tau_{2}$ is the normalized trace on $M_{2}(\mathbf{C}), \nu$ is a positive measure on $\left[0, \frac{\pi}{2}\right], a_{1}, a_{2}, b_{1}, b_{2} \geq 0$ and $|\nu|+a_{1}+a_{2}+b_{1}+b_{2}=1$. By Example 2.8 of [9], the distribution of $p q p$ in $p \mathscr{M} p$ has no atoms, which implies that $|\nu|=1$ and $\nu$ has no atoms.

REMARK 3.3. In the right-hand side of (10), let

$$
x=\left(\begin{array}{ll}
0 & 0 \\
1 & 0
\end{array}\right)=\operatorname{pol}((1-p) q p),
$$

where "pol" means "polar part of." Then $x$ is a partial isometry from $p$ to $1-p$ and $\mathscr{M}$ is generated by $p q p$ together with $x$. Let $y=\operatorname{pol}((1-q) p q)$. Then $y$ is a partial isometry from $q$ to $1-q$. Let

$$
w=\left(\begin{array}{rr}
\cos \theta & -\sin \theta \\
\sin \theta & \cos \theta
\end{array}\right) .
$$

Then $w$ is unitary and $w p w^{*}=q, w x w^{*}=y$.

Definition 3.4. Let $\left(S_{l}\right)_{l \in I}$ be subsets of a unital algebra $A \ni 1$. A nontrivial traveling product in $\left(S_{l}\right)_{l \in I}$ is a product $a_{1} a_{2} \cdots a_{n}$ such that $a_{j} \in S_{l_{j}}(1 \leq j \leq n)$ and $\imath_{1} \neq l_{2} \neq l_{3} \neq \cdots \neq l_{n}$. The trivial traveling product is the identity element $1 . \Lambda\left(\left(S_{l}\right)_{l \in I}\right)$ denotes the set of all traveling products in $\left(S_{l}\right)_{l \in I}$, including the trivial one. If $|I|=2$, we will often call traveling products alternating products.

Theorem 3.5. Let $A$ and $B$ be finite von Neumann algebras (with specified faithful traces-see Remark 3.1). Then

(i) $\left(A \otimes L\left(\mathbf{Z}_{2}\right)\right) *\left(B \otimes L\left(\mathbf{Z}_{2}\right)\right) \cong(A * A * B * B * L(\mathbf{Z})) \otimes M_{2}$,

(ii) $\left(A \otimes M_{2}\right) *\left(B \otimes L\left(\mathbf{Z}_{2}\right)\right) \cong\left(A * B * B * L\left(\mathbf{F}_{2}\right)\right) \otimes M_{2}$,

(iii) $\left(A \otimes M_{2}\right) *\left(B \otimes M_{2}\right) \cong\left(A * B * L\left(\mathbf{F}_{3}\right)\right) \otimes M_{2}$. 
Proof. Let $\mathscr{M}$ be the von Neumann algebra on the left-hand side of (i) with trace $\tau$. It will be notationally convenient to identify $A$ with $A \otimes 1 \subseteq \mathscr{M}$ and $B$ with $B \otimes 1 \subseteq \mathscr{M}$. Let $p$ and $q$ be projections of trace $\frac{1}{2}$ contained in the copy of $1 \otimes L\left(\mathbf{Z}_{2}\right)$ that commute with $A$ and respectively $B$. Let $\mathscr{N}_{0}=\{p, q\}^{\prime \prime} \cong L\left(\mathbf{Z}_{2}\right) * L\left(\mathbf{Z}_{2}\right)$, and let $x, y, w \in \mathscr{N}_{0}$ be as in Remark 3.3. Then

$$
p \mathscr{M} p=\left(\{p q p\} \cup p A \cup x^{*} A x \cup w^{*} q B w \cup w^{*} y^{*} B y w\right)^{\prime \prime} .
$$

We claim moreover that $\left\{\{p q p\}, p A, x^{*} A x, w^{*} q B w, w^{*} y^{*} B y w\right\}$ is a free family in $p \mathscr{M} p$, which then clearly implies (i).

Let us first show that $\left\{\{p q p\}, p A, x^{*} A x\right\}$ is free in $p \mathscr{M} p$. Let $g_{k}=(p q p)^{k}-2 \tau\left((p q p)^{k}\right) p \quad(k \geq 1)$. To show freeness means to show that a nontrivial traveling product in $\left\{g_{k} \mid k \geq 1\right\}, p \AA$ and $x^{*} \stackrel{\circ}{A} x$ has trace zero. Regrouping gives a traveling product in $\Omega_{0}=$ $\left\{x, x^{*}\right\} \cup\left\{g_{k}, x g_{k}, g_{k} x^{*}, x g_{k} x^{*} \mid k \geq 1\right\}$ and $\stackrel{\circ}{A}$. Let $a=p-\frac{1}{2}$, $b=q-\frac{1}{2}$. Then $\mathscr{N}_{0}=\{a, b\}^{\prime \prime}$, and $\operatorname{span} \Lambda(\{a\},\{b\})$ is a dense *-subalgebra of $\mathscr{N}_{0}$. Note that $\Omega_{\circ} \subset \mathscr{N}_{0}$, so that by the Kaplansky Density Theorem, any $z \in \Omega_{0}$ is the s.o.-limit of a bounded sequence in span $\Lambda(\{a\},\{b\})$. Note also that since $a$ and $b$ are free and each has trace zero, the trace of an element of $\operatorname{span} \Lambda(\{a\},\{b\})$ is equal to the coefficient of 1 . Since $\tau(z)=0$, we may choose that approximating sequence in $\operatorname{span} \Lambda(\{a\},\{b\})$ so that each coefficient of 1 equals zero. Moreover, since also $\tau(p z)=0$, we may also insist that each coefficient of $a$ be zero, i.e. we have a bounded approximating sequence for $z$ of elements of $\operatorname{span}(\Lambda(\{a\},\{b\}) \backslash\{1, a\})$. We must now only show that a nontrivial alternating product in $\Lambda(\{a\},\{b\}) \backslash\{1, a\}$ and $\stackrel{\circ}{A}$ has trace zero. Regrouping gives a nontrivial alternating product in $\{a\} \cup \stackrel{\circ}{A} \cup a \stackrel{\circ}{A}$ and $\{b\}$, which by freeness has trace zero.

Let $\mathscr{N}_{1}=\left(A \cup \mathscr{N}_{0}\right)^{\prime \prime}$, and let us show that $\left\{q w \mathscr{N}_{1} w^{*}, q B, y^{*} B y\right\}$ is free in $q \mathscr{M} q$, which will complete the proof of (i). We show that a nontrivial traveling product in $w \mathscr{N}_{1} w^{*}, q \stackrel{\circ}{B}$ and $y^{*} \stackrel{\circ}{B} y$ has trace zero. Regrouping gives a traveling product in $\Omega_{1}=\left\{y, y^{*}\right\} \cup$ $q w \stackrel{\circ}{N}_{1} w^{*} \cup y w \stackrel{\circ}{N}_{1} w^{*} \cup w \stackrel{\circ}{N}_{1} w^{*} y^{*} \cup y w \stackrel{\circ}{N}_{1} w^{*} y^{*}$ and $\stackrel{\circ}{B}$. Now $\Omega_{1} \subset$ $\mathscr{N}_{1}, \operatorname{span} \Lambda(\{a\} \cup \stackrel{\circ}{A} \cup a \AA,\{b\})$ is a dense *-subalgebra of $\mathscr{N}_{1}$ and $\tau(z)=\tau(q z)=0 \forall z \in \Omega_{1}$, so that as above, each $z \in \Omega_{1}$ is the s.o.-limit of a bounded sequence in

$$
\operatorname{span}(\Lambda(\{a\} \cup \stackrel{\circ}{A} \cup a \stackrel{\circ}{A},\{b\}) \backslash\{1, b\}) .
$$


So it suffices to show that a nontrivial alternating product in $\operatorname{span}(\Lambda(\{a\} \cup \stackrel{\circ}{A} \cup a \stackrel{\circ}{A},\{b\}) \backslash\{1, b\})$ and $\stackrel{\circ}{B}$ has trace zero. Regrouping gives a nontrivial alternating product in $\{a\} \cup \stackrel{\circ}{A} \cup a \stackrel{\circ}{A}$ and $\{b\} \cup \stackrel{\circ}{B} \cup b \stackrel{\circ}{B}$, which by freeness has trace zero.

Now we prove (ii). Let $\mathscr{M}$ be the von Neumann algebra on the left-hand side of (ii), and let $\tau$ be its trace. We will identify $A$ with $A \otimes 1$ and $B$ with $B \otimes 1$ as in the proof of (i). Let $p$ be a projection in $1 \otimes M_{2}$ (commuting with $A$ ) of trace $\frac{1}{2}$ and $q$ a projection in $1 \otimes L\left(\mathbf{Z}_{2}\right)$ (commuting with $B$ ) of trace $\frac{1}{2}$. Let $\mathscr{N}_{0}=\{p, q\}^{\prime \prime}$ and let $x, y, w, z, b \in \mathscr{N}_{0}$ be as in the proof of (i). Let $u \in 1 \otimes M_{2}$ be a partial isometry from $p$ to $1-p$. Then

$$
p \mathscr{M} p=\left(\left\{p q p, x^{*} u\right\} \cup p A \cup w^{*} q B w \cup w^{*} y^{*} B y w\right)^{\prime \prime},
$$

and we shall show that $x^{*} u$ is a Haar unitary (i.e. a unitary such that $\left(x^{*} u\right)^{n}$ has trace zero $\left.\forall n \in \mathbf{Z} \backslash\{0\}\right)$ and that $\left\{\{p q p\},\left\{x^{*} u\right\}, p A\right.$, $\left.w^{*} q B w, w^{*} y^{*} B y w\right\}$ is $*$-free in $p \mathscr{M} p$. This will in turn prove (ii). For $n>0, r=\left(x^{*} u\right)^{n}$ is a nontrivial alternating product in $\left\{x^{*}\right\}$ and $\{u\}$, and $x^{*}$ is the s.o.-limit of a bounded sequence in $\operatorname{span}(\Lambda(\{a\},\{b\}) \backslash\{1, a\})$, so to show $\tau(r)=0$ it suffices to show that a nontrivial alternating product in $\operatorname{span}(\Lambda(\{a\},\{b\}) \backslash\{1, a\})$ and $\{u\}$ has trace zero. Regrouping gives a nontrivial alternating product in $\{a, u\}$ and $\{b\}$, which by freeness has trace zero. Hence we have shown that $x^{*} u$ is a Haar unitary in $p \mathscr{M} p$.

Now we show that $x^{*} u$ and $p q p$ are $*$-free in $p \mathscr{M} p$. Let $g_{k}$ $(k \geq 1)$ be as in the proof of (i). It suffices to show that a nontrivial alternating product in $\left\{\left(x^{*} u\right)^{n} \mid n \in \mathbf{Z} \backslash\{0\}\right\}$ and $\left\{g_{k} \mid k \geq 1\right\}$ has trace zero. Regrouping gives an alternating product in $\Omega_{0}$ and $\left\{u, u^{*}\right\}$, where $\Omega_{0}$ is as in the proof of (i), which, proceeding as we did above, we see has trace zero. Similarly, we can show that letting $\widetilde{\mathscr{N}_{0}}=\left\{p q p, x^{*} u\right\}^{\prime \prime},\{\widetilde{\mathscr{N}}, p A\}$ is free in $p \mathscr{M} p$, and that letting $\widetilde{\mathscr{N}_{1}}=\left(\widetilde{\mathscr{N}_{0}} \cup A\right)^{\prime \prime},\left\{w^{*} \widetilde{\mathscr{N}_{1}} w, q B, y^{*} B y\right\}$ is free in $q \mathscr{M} q$, thus proving (ii).

To prove (iii), let $p$ and $u$ in $1 \otimes M_{2}$ commuting with $A$ be as above, let $q \in 1 \otimes M_{2}$ commuting with $B$ be a projection of trace $\frac{1}{2}$ and $v \in 1 \otimes M_{2}$ commuting with $B$ a partial isometry from $q$ to $1-q$. Let $x, y, w \in \mathscr{N}_{0}=\{p, q\}^{\prime \prime}$ be as above. Then we similarly show that $x^{*} u$ and $y^{*} v$ are Haar unitaries and that $\left\{\{p q p\},\left\{x^{*} u\right\}, p A,\left\{w^{*} y^{*} v w\right\}, w^{*} q B w\right\}$, is $*$-free in $p \mathscr{M} p$ (and notice that these taken together generate $p \mathscr{M} p$ ), which proves (iii). 
COROLLARY 3.6. Let $R$ and $\widetilde{R}$ be copies of the hyperfinite $\mathrm{II}_{1}$ factor. Then

$$
R * \widetilde{R} \cong R * L(\mathbf{Z}),
$$

with an isomorphism which when restricted is the identity map from $R$ to $R$.

Proof. Write $R=(p R p) \otimes M_{2}$ and $\widetilde{R}=(\tilde{p} \tilde{R} \tilde{p}) \otimes M_{2}$, where $p$ and $\tilde{p}$ are projections of trace $\frac{1}{2}$ in $R$ and respectively $\widetilde{R}$. Then by (iii) and the proof of (iii),

$$
p(R * \widetilde{R}) p \cong(p R p) *(\tilde{p} \widetilde{R} \tilde{p}) * L\left(\mathbf{F}_{3}\right),
$$

and the isomorphism when restricted to $p R p \subset p(R * \widetilde{R}) p$ is the identity map from $p R p$ to $p R p$. Similarly, writing also $L(\mathbf{Z}) \cong$ $L(\mathbf{Z}) \otimes L\left(\mathbf{Z}_{2}\right)$, we have from (ii) and the proof of (ii) that

$$
p(R * L(\mathbf{Z})) p \cong(p R p) * L\left(\mathbf{F}_{4}\right),
$$

and the isomorphism, when restricted to $p R p \subset p(R * L(\mathbf{Z})) p$, is the identity map from $p R p$ to $p R p$. Considering the isomorphism (3), we get an isomorphism from $p(R * \widetilde{R}) p$ to $p(R * L(\mathbf{Z})) p$ which when restricted is the identity map on $p R p$. Now tensor with $M_{2}$.

\section{The addition formula for free products.}

$$
\text { THEOREM 4.1. } L\left(\mathbf{F}_{r}\right) * L\left(\mathbf{F}_{r^{\prime}}\right)=L\left(\mathbf{F}_{r+r^{\prime}}\right) \text { for } 1<r, r^{\prime} \leq \infty \text {. }
$$

Proof. (Please see the comments at the end of the introduction.) In a W*-probability space $(\mathscr{M}, \tau)$ where $\tau$ is a trace, let $R$ and $\widetilde{R}$ be copies of the hyperfinite $\mathrm{II}_{1}$-factor and let $\nu=\left\{X^{t} \mid t \in T\right\}$ be a semicircular family such that $\{R, \widetilde{R}, \nu\}$ is free. Let

$$
\begin{aligned}
L\left(\mathbf{F}_{r}\right) & =\mathscr{A}=\left(R \cup\left\{p_{s} X^{s} p_{s} \mid s \in S\right\}\right)^{\prime \prime}, \\
L\left(\mathbf{F}_{r^{\prime}}\right) & =\mathscr{B}=\left(\widetilde{R} \cup\left\{q_{s} X^{s} q_{s} \mid s \in S^{\prime}\right\}\right)^{\prime \prime},
\end{aligned}
$$

where $S$ and $S^{\prime}$ are disjoint subsets of $T, p_{s} \in R, q_{s} \in \widetilde{R}$ are projections and where $1+\sum_{s \in S} \tau\left(p_{s}\right)^{2}=r, 1+\sum_{s \in S^{\prime}} \tau\left(q_{s}\right)^{2}=r^{\prime}$. Then $\mathscr{A}$ and $\mathscr{B}$ are free in $(\mathscr{M}, \tau)$, so

$$
L\left(\mathbf{F}_{r}\right) * L\left(\mathbf{F}_{r^{\prime}}\right) \cong \mathscr{N}=\left(R \cup \widetilde{R} \cup\left\{p_{s} X^{s} p_{s} \mid s \in S\right\} \cup\left\{q_{s} X^{s} q_{s} \mid s \in S^{\prime}\right\}\right)^{\prime \prime} .
$$

By Corollary 3.6, there exists a semicircular element $Y \in \mathscr{N}_{0}=$ $(R \cup \widetilde{R})^{\prime \prime}$ such that $R$ and $\{Y\}$ are free and together they generate 
$\mathscr{N}_{0}$. Moreover, for $s \in S^{\prime}$ let $U_{s} \in \mathscr{N}_{0}$ be a unitary such that $U_{s} q_{s} U_{s}^{*}=f_{s} \in R$. Then

$$
\mathscr{N}=\left(R \cup\{Y\} \cup\left\{p_{s} X^{s} p_{s} \mid s \in S\right\} \cup\left\{f_{s}\left(U_{s} X^{s} U_{s}^{*}\right) f_{s} \mid s \in S^{\prime}\right\}\right)^{\prime \prime} .
$$

To prove the theorem, it suffices to observe that $\left\{R,\{Y\},\left(\left\{X^{s}\right\}\right)_{s \in S}\right.$, $\left.\left(\left\{U_{s} X^{s} U_{s}^{*}\right\}\right)_{s \in S^{\prime}}\right\}$ is free in $\mathscr{M}$.

Let us recall [4] that the fundamental group of a $\mathrm{II}_{1}$-factor $\mathscr{M}$ is defined to be the set of positive real numbers $\gamma$ such that $\mathscr{M}_{\gamma} \cong \mathscr{M}$. Murray and von Neumann [4] showed that the fundamental group of the hyperfinite $\mathrm{II}_{1}$-factor is $\mathbf{R}_{+}$, and recently Rădulescu [5] has shown that the fundamental group of $L\left(\mathbf{F}_{\infty}\right)$ is also $\mathbf{R}_{+}$. A. Connes [1] has shown that the fundamental group of $L(G)$ where $G$ is a group with property $T$ of Kazhdan must be countable, but no other examples are known for fundamental groups of $\mathrm{II}_{1}$-factors.

Equation (2) shows that the isomorphism question for (interpolated) free group factors is equivalent to the fundamental group question. Combined with the addition formula for free prdoucts, we now see that we must have one of two extremes.

\section{COROLLARY 4.2. We must have either}

(I) $L\left(\mathbf{F}_{r}\right) \cong L\left(\mathbf{F}_{r^{\prime}}\right)$ for all $1<r, r^{\prime}<\infty$ and the fundamental group of $L\left(\mathbf{F}_{r}\right)$ is $\mathbf{R}_{+}$for all $1<r<\infty$, or

(II) $L\left(\mathbf{F}_{r}\right) \not L\left(\mathbf{F}_{r^{\prime}}\right)$ for all $1<r<r^{\prime}<\infty$ and the fundamental group of $L\left(\mathbf{F}_{r}\right)$ is $\{1\}$ for all $1<r<\infty$.

Proof. Using formulas (1) and (2) we can show that if $L\left(\mathbf{F}_{r}\right)=$ $L\left(\mathbf{F}_{r^{\prime}}\right)$ for some $r \neq r^{\prime}$, then we have $L\left(\mathbf{F}_{r}\right)=L\left(\mathbf{F}_{r^{\prime \prime}}\right)$ for $r^{\prime \prime}$ in some open interval, hence that the fundamental group of $L\left(\mathbf{F}_{r}\right)$ contains an open interval, thus is all of $\mathbf{R}_{+}$.

Acknowledgments. I would like to thank Dan Voiculescu, my advisor, for helpful discussions and for suggesting I look at free products such as $M_{2}(\mathbf{C}) * M_{2}(\mathbf{C})$.

\section{REFERENCES}

[1] A. Connes, A factor of type $\mathrm{II}_{1}$ with countable fundamental group, J. Operator Theory, 4 (1980), 151-153.

[2] K. Dykema, On certain free product factors via an extended matrix model, J. Funct. Anal., 112 (1993), 31-60.

[3] _ Free products of hyperfinite von Neumann algebras and free dimension, Duke Math. J., 69 (1993), 97-119. 
[4] F. J. Murray and J. von Neumann, Rings of operators. IV, Ann. of Math., 44 (1943), 716-808.

[5] F. Rădulescu, The fundamental group of the von Neumann algebra of a free group with infinitely many generators is $\mathbf{R}_{+} \backslash\{0\}$, J. Amer. Math. Soc., 5 (1992), 517-532.

[6] _ Stable isomorphism of the weak closure of free group convolution algebras, Inst. Hautes Études Sci. Publ. Math., December, 1991, preprint.

[7] __, Random matrices, amalgamated free products and subfactors of the von Neumann algebra of a free group, Inst. Hautes Études Sci. Publ. Math., December, 1991, preprint.

[8] D. Voiculescu, Symmetries of some reduced free product $C^{*}$-algebras, Operator Algebras and Their Connections with Topology and Ergodic Theory, Lecture Notes in Math., vol. 1132, Springer-Verlag, 1985, pp. 556-588.

[9] _ Multiplication of certain non-commuting random variables, J. Operator Theory, 18 (1987), 223-235.

[10] , Noncommutative random variables and spectral problems in free product $C^{*}$-algebras, Rocky Mountain J. Math., 20 (1990), 263-283.

[11] _ Circular and semicircular systems and free product factors, Operator Algebras, Unitary Representations, Enveloping Algebras, and Invariant Theory, Progress in Mathematics, vol. 92, Birkhäuser, Boston, 1990.

[12] L Limit laws for Random matrices and free products, Invent. Math., 104 (1991), 201-220.

[13] - Free non-commutative random variables, random matrices and the $\mathrm{II}_{1}$ factors of free groups, Quantum Probability and Related Topics VI, L. Accardi, ed., World Scientific, Singapore, 1991, pp. 473-487.

Received April 13, 1992 and in revised form August 21, 1992. Studies and research supported by the Fannie and John Hertz Foundation.

UNIVERSITY OF CALIFORNIA

BERKELEY, CA 94720

E-mail address: dykema@math.berkeley.edu 



\section{CONTENTS}

P. Ahern and W. Cohn, Weighted maximal functions and derivatives of invariant Poisson integrals of potentials $\ldots, \ldots, \ldots, \ldots, \ldots, \ldots, \ldots, \ldots, \ldots, \ldots$

V. Ancona, T. Peternell, and J. A. Wiśniewski, Fano bundles and splitting theorems

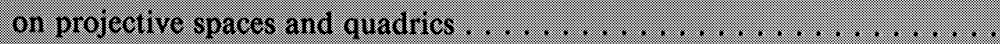

G. M. Beffa, A transverse structure for the Lie-Poisson bracket on the dual of the

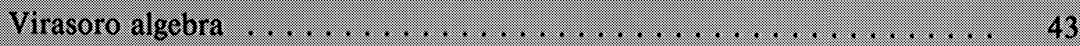

C. Biasi, On ambiental bordism . . . . . . . . . . . . . . . . . 73

P. Debes and M. D. Fried, Nonrigid constructions in Galois theory . . . . . . 81

K. Dykema, Interpolated free group factors . . . . . . . . . . . . . 123

S.-A. Kim and D. Minda, Two-point distortion theorems for univalent functions 137

X. Mo, Value distribution of the Gauss map and the total curvature of complete minimal surface in $R^{m} \ldots \ldots \ldots \ldots \ldots \ldots \ldots \ldots \ldots \ldots$

Z. Shen, On complete Riemannian manifolds with collapsed ends . . . . . . . . 175

F. Zheng, Curvature characterization of certain bounded domains of holomorphy 183

S. Zhu, The classification of complete locally conformally flat manifolds of nonnegative Ricci curvature . . . . . . . . . . . . . . . . . . . . . . 189 


\section{PACIFIC JOURNAL OF MATHEMATICS}

Volume $163 \quad$ No. $1 \quad$ March 1994

Weighted maximal functions and derivatives of invariant Poisson integrals $\quad 1$ of potentials

PATRICK Robert AHERn and WiLliam S. COHN

Fano bundles and splitting theorems on projective spaces and quadrics

VincENZO ANCONA, ThOMAS PETERnELL and JAROSLAW

WISNIEWSKI

A transverse structure for the Lie-Poisson bracket on the dual of the

Virasoro algebra

GLORIA MARI BEFFA

On ambiental bordism

CARLOS BIASI

Nonrigid constructions in Galois theory

PIERRE DÈbes and Michael Fried

Interpolated free group factors

KENNETH JAY DYKEMA

Two-point distortion theorems for univalent functions

SEONG-A KIM and C. DAVID (CARL) MINDA

Value distribution of the Gauss map and the total curvature of complete minimal surface in $R^{m}$

XIAOKANG Mo

On complete Riemannian manifolds with collapsed ends

ZHONGMIN SHEN

Curvature characterization of certain bounded domains of holomorphy

FANGYANG ZHENG

The classification of complete locally conformally flat manifolds of nonnegative Ricci curvature

SHUN-HUi ZHU 